Research Article

\title{
Research on the Cutting Principle and Tool Design of Gear Skiving Based on the Theory of Conjugate Surface
}

\author{
Peng Wang $\mathbb{D}$, Jingcai Li, and Lin Han \\ Tianjin Key Laboratory of High Speed Cutting and Precision Machining, Tianjin University of Technology and Education, \\ Tianjin 300222, China \\ Correspondence should be addressed to Peng Wang; tjujxwp@126.com
}

Received 3 August 2020; Revised 31 December 2020; Accepted 13 March 2021; Published 27 March 2021

Academic Editor: Kai Zhang

Copyright (c) 2021 Peng Wang et al. This is an open access article distributed under the Creative Commons Attribution License, which permits unrestricted use, distribution, and reproduction in any medium, provided the original work is properly cited.

Tool design is one of the key factors that restrict the development of gear skiving technology since the design principle does not correspond to the cutting principle. The existing skiving tool cannot achieve ideal machining accuracy and reasonable cutting angles. In view of this, some research has been done in this paper. Firstly, the skiving principle is investigated essentially according to the skiving motions. Then, the principle of tool design is analyzed based on the theory of conjugate surface, and a new tool design method is proposed to match the skiving principle. For this, all the skiving patterns for various kinds of workpieces are enumerated and summarized to abstract a normalized skiving model. Based on this, the mathematical model of the conjugate surface is then derived to lay the foundation for tool design. Then, the design methods of cutting edge, rake face, and flank face are proposed. An example is presented at last, and the cutting simulation is conducted. The result proves that the proposed methods are correct and valid. The theoretical research in this paper could promote the improvement of skiving tools.

\section{Introduction}

Gear skiving $[1,2]$ is a new processing technology which has been realized just from the beginning of the 21 st century with the development of numerical control technology. It has high efficiency and high precision in cutting many kinds of gears no matter involute gears or other special gears with noninvolute surfaces. However, it is a pity that this technology has not been widely used in engineering although it has been developed for many years. One of the important reasons is that tool design cannot meet the requirement.

As we all know, tool design methods need support from corresponding theory. At present, the skiving tool is basically designed according to the envelope theory. The common slotting tool is even directly used for gear skiving in some cases [3]. The existing skiving tools usually have the following problems in engineering: (1) the machining accuracy of the two sides of the workpiece is inconsistent $[4,5]$. (2) Interference is easy to occur in the cutting process $[6,7]$. (3) The cutting edge has theoretical shape error and the machining accuracy after tool regrinding would be obviously different from that before regrinding $[8,9]$. (4) The tool wear would be fast and uneven if the tool and skiving parameters $[10,11]$ were unreasonably set. In order to solve these problems, some scholars have done much rewarding work on the design of skiving tool. Among the latest research, the theory of conjugate surface $[12,13]$ is the most commonly used. Based on this theory, some error-free design methods $[14,15]$ for the cutting edge have been proposed. In addition, Guo et al. [16] proposed a novel skiving tool with multiple subblades on the rake face to improve the tool life. Guo et al. [17] investigated the multiple-side-feeds technique and found that this technique is still futile for the wear reducing of the up-recess edge. The, they proposed an improved technique according to the edge shape. In addition to this, some research shows that the working rake and relief angles have great influence on the cutting performance of the tool, such as cutting force [18]. Moriwaki et al. [19] studied the change rule of working rake angle and working relief angle and found that the working rake angle was likely to be negative. Tapoglou [20] established a novel simulation model to predict the skiving chips. Then, the influence of 
tool and machining parameters on chip geometry was analyzed, which provided a way to optimize the tool. Tachikawa et al. [21] calculated the cutting force in gear skiving by a geometrical method and then proposed a method to reduce vibrations. These studies provide references for the design of skiving tool. However, the above problems have not been fully solved due to the lack of general design theory and method that fully adapt to the skiving principle.

The main structures of a skiving tool include cutting edge, rake face, and flank face. Among them, the cutting edge is the most important factor affecting the machining accuracy. During the design process, it is necessary to ensure that the shape of the cutting edge is correct without overcutting or undercutting and easy to achieve the required accuracy when tool manufacturing or regrinding. For this, the cutting edge is picked from the conjugate surface based on the essential principle of gear skiving to ensure that it is error-free. Meanwhile, the shape of the cutting edge is designed to be smooth and simple to the greatest extent. Then, its manufacturing accuracy can be ensured further by five-axis tool grinding technology [22]. In this paper, the skiving principle is investigated in Section 2. And then, a new tool design method is proposed in Section 3 to achieve not only error-free cutting edge but also tool faces with reasonable and even working angles. A unified mathematical motion model for tool design is established in Section 4. As the core content of this article, the mathematical model of the conjugate surface is derived in Section 5. A tool design example is presented in Section 6, and a cutting simulation is done to prove that the proposed tool design method is correct. Conclusions are enclosed in Section 7.

\section{Skiving Principle}

Two rotary motions and one linear motion are needed to realize gear skiving, that is, the rotation of the workpiece, the rotation of the cutter, and the linear motion of the workpiece (or the cutter) along the axial direction of the workpiece. When skiving, the tool axis has an intersection angle with the workpiece axis. In order to make the cutting amount reasonable, the total cutting layer is divided into several times of radial feeding. Each radial feeding is realized by adjusting the vertical distance between the tool axis and the workpiece axis. Because the shape of the finished tooth surface is mainly formed by the last radial feeding, this paper only considers the last radial feeding as tool design.

The main motions of gear skiving can be simply shown as Figure 1. The tool rotates clockwise along its axis and the workpiece rotates anticlockwise along its own axis. It is imagined that a point $M$ on the cutting edge of the tool moves from position 5 to position 1 and coincides with point $N$ which is on the workpiece. At this time, the directions of the velocities at these two points are shown in Figure 1. Due to the intersection angle between the axes, the relative velocity has a component along the axial direction of the workpiece. This relative motion brings about the effect of material excision. In order to obtain correct tooth profile, the trajectory of point $M$ relative to the workpiece should be tangent to the theoretical tooth surface, as shown in Figure 2.

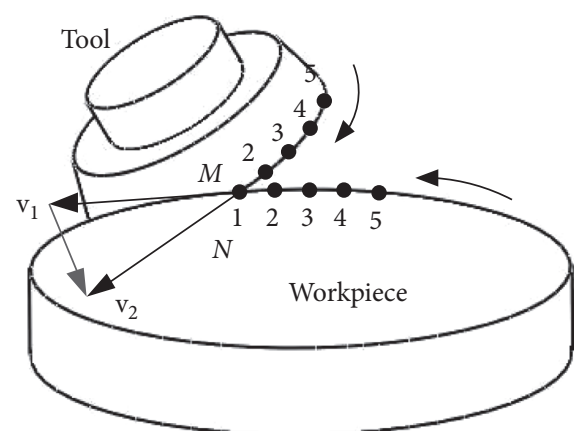

Figure 1: Synchronous motions of skiving.

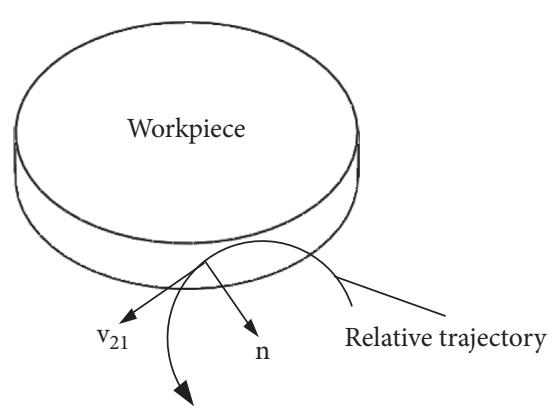

FIGURE 2: Trajectory of a point on cutting edge.

At the tangency point, the relative velocity $\mathbf{v}_{21}$ (or called cutting speed) is located in the tangent plane of the tooth surface. Therefore, the cutting speed $\mathbf{v}_{21}$ is perpendicular to the normal vector $\mathbf{n}$ of the tooth surface. That is to say, the points on the cutting edge satisfy the conjugate condition. Therefore, the ideal cutting edge should be located on the conjugate surface of the tooth surface.

As shown in Figure 3, in the cutting process, the cutting edge continuously sweeps relative to the workpiece to remove material. According to the conjugation theory, the cutting edge, as a curve on the conjugate surface, would contact with a certain curve on the tooth surface. This curve is exactly the theoretical tooth profile. After edge-sweeping, a complete tooth profile would be shaped on the workpiece. This process is called conjugate rotary cutting.

Obviously, the profile forming principle of gear skiving is different from that of gear slotting or gear hobbing as shown in Figure 4. The main cutting motion of gear slotting is the reciprocating linear motion of the slotting tool, and only part of the tooth profile can be shaped at each time. The whole tooth profile is formed section by section with the enveloping motions. By contrast, the conjugate rotary cutting can integrally form the tooth profile directly by the main cutting motions, other than enveloping motions. Therefore, the profile accuracy of the workpieces machined by gear skiving is higher than that of gear slotting or hobbing in theory.

\section{Design Principle of Skiving Tool}

According to the cutting principle, the cutting edge should be located on the conjugate surface of the theoretical tooth surface. In tool design, the conjugate surface is calculated first, 


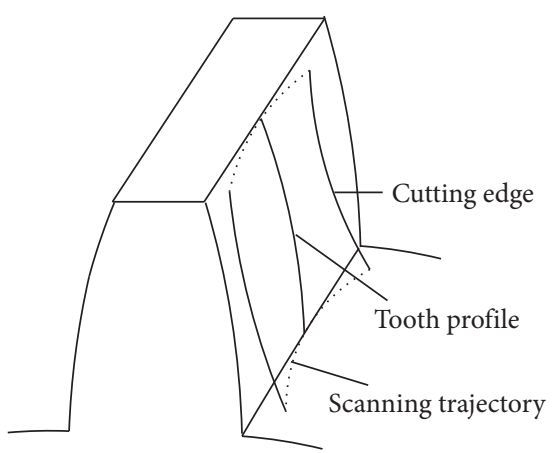

FIGURE 3: Sketch of the scanning trajectory of the cutting edge.

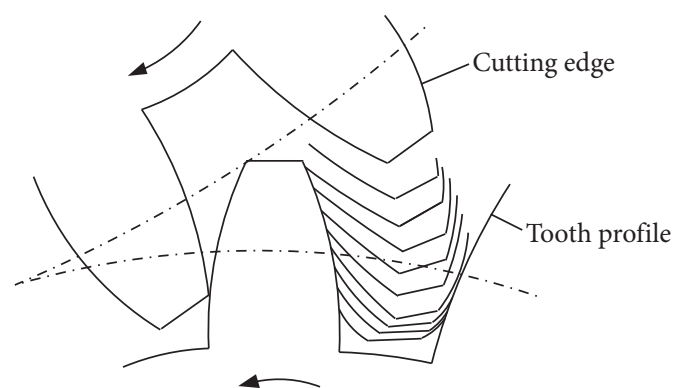

FIgURE 4: Tooth formation principle of the enveloping method.

and then the cutting edge is selected from the conjugate surface. The specific selection method should fit with the characteristic of the workpiece. For instance, the cutting edge for involute gear can be obtained by intersecting of an auxiliary plane and the conjugate surface to simplify its shape.

The rake face plays the role of chip removal. Accordingly, the rake angle has much influence on cutting force and tool wear. In order to make the cutting process smooth, it should be ensured that the working rake angle is consistent. For this, a kind of free-form surface may be adopted to construct rake face. However, this kind of rake face would be complex and difficult to be manufactured. In view of this, the rake face can also be constructed by a simple regular surface, such as a plane, in engineering application.

The flank face is adjacent to the machined surface of the workpiece in the skiving process. Therefore, the basic requirement is that the flank face has suitable relief angles to reduce the friction between the machined tooth surface and the tool. Furthermore, in order to ensure the consistency of machining accuracy after tool regrinding, the flank face is constructed by the ideal cutting edges which would be obtained correspondingly to every time of regrinding.

To sum up, the cutting edge is designed firstly based on conjugate principle. The tool faces are constructed over the cutting edge aiming at reasonable working angles and constant cutting performance. The design process is shown in Figure 5.

\section{Normalized Motion Model of Gear Skiving}

4.1. Position and Motion Relationship between Tool and Workpiece. As the basis of tool design, a standardized motion model of skiving is established in this section by summarizing the relationship between tool and different

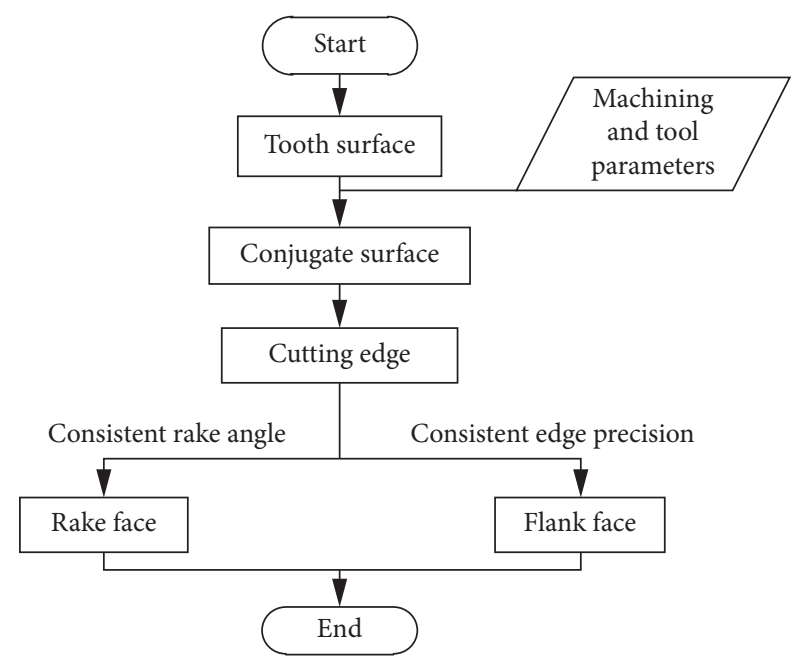

FIgURE 5: Design flow of skiving tool.

types of workpiece. In this paper, only the workpiece with internal right-lateral tooth is analyzed as an example, due to space limitation.

As shown in Figures 6-8, $\gamma$ means the angle between the tool axis and the workpiece axis, called shaft angle. $\beta_{1}$ means the helix angle of the workpiece. $\beta_{2}$ means the helix angle of the tool. $\omega_{1}$ means the angular speed of the workpiece, and $\omega_{2}$ means the angular speed of the tool. $f$ means the axial feed rate of the workpiece. In order to achieve correct cutting, at the cutting position, the direction of the tool tooth is required to be consistent with that of the workpiece slot. Under this requirement, there are three choices for the shaft angle: (1) the tool axis swings to the left, and $\gamma>\beta_{1}$, as shown in Figure 6; (2) the tool axis swings to the left, and $\gamma<\beta_{1}$, as shown in Figure 7; and (3) the tool axis swings to the right, as shown in Figure 8.

The characteristics of the helix angle of the tool in the above three cases are as follows: in Case 1, the tool is leftlateral with the helix angle $\beta_{2}<\gamma$; in Case 2 , the tool is right-lateral with the helix angle $\beta_{2}<\beta_{1}$; in Case 3 , the tool is right-lateral with the helix angle $\beta_{2}>\gamma$. Among them, Case 3 would make the tool helix angle too large, which is bad for tool manufacturing, while Cases 1 and 2 have reasonable tool helix angles. Comparing Case 1 with Case 2 , it can be seen that the shaft angle in Case 1 would be obviously larger than that in Case 2 under the same tool helix angle. For workpiece with helix angle $\beta_{1}<20^{\circ}$, Case 1 should be adopted to obtain larger shaft angle, which can improve cutting speed. For workpiece with helix angle $\beta_{1} \geq 20^{\circ}$, it is suitable to adopt Case 2 , which can divide the relatively large workpiece helix angle into the shaft angle and the tool helix angle, so as to obtain balanced and reasonable angles.

By the same way, the other cases can also be analyzed. To sum up, the shaft angle should be set as shown in Table 1 for workpieces with different kinds of helix angle.

It should be pointed out that the rotation directions of the tool and the workpiece are determined only by the swing direction of the tool axis. Following the direction of view shown in Figures 6-8, if the swing direction of the tool axis is 


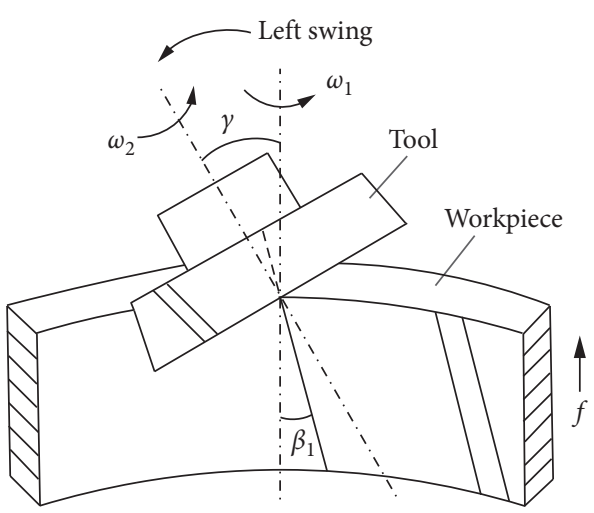

Figure 6: Case 1 of skiving settings.

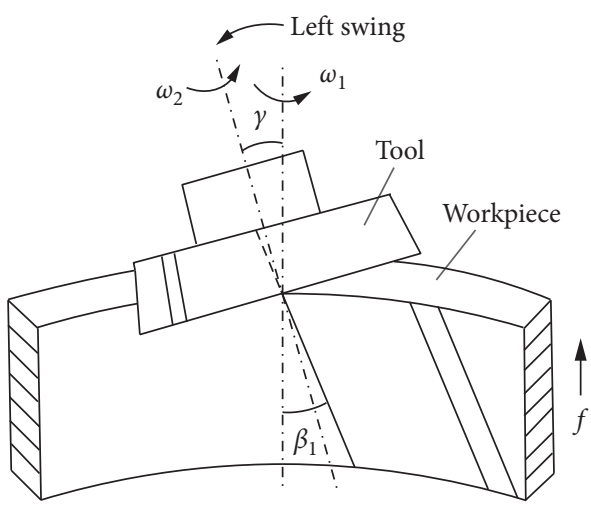

FIgURE 7: Case 2 of skiving settings.

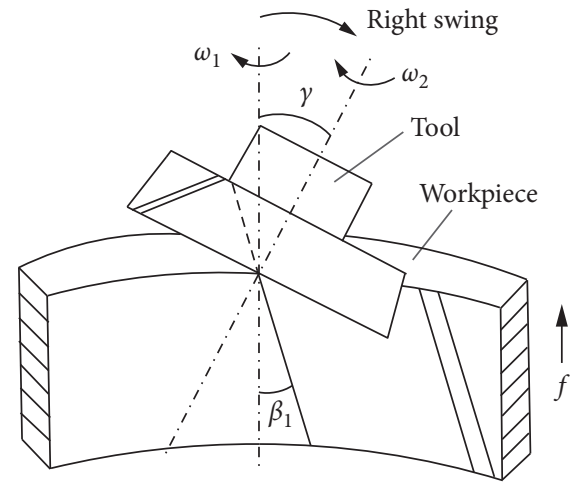

FIGURE 8: Case 3 of skiving settings.

left, both the tool and the workpiece should rotate anticlockwise. If the swing direction of the tool axis is right, both the tool and the workpiece should rotate clockwise. Only in this way can the cutting process be carried out.

4.2. Abstract Motion Model of Skiving. Based on the analysis in Section 4.1, a normalized motion model can be abstracted to describe the essence of skiving. As shown in Figure 9, the difference between skiving internal tooth and skiving external tooth is embodied in the different center distance and the opposite rotation direction of the tool. All the cases can be abstracted in the motion model as shown in Figure 10 just
TABLE 1: Shaft angle setting and helical angle of the workpiece.

\begin{tabular}{lc}
\hline Workpiece helix angle $\beta_{1}$ & Shaft angle $\gamma$ \\
\hline Right-lateral and $\beta_{1}<20^{\circ}$ & Left swing and $\gamma>\beta_{1}$ \\
Right-lateral and $\beta_{1} \geq 20^{\circ}$ & Left swing and $\gamma<\beta_{1}$ \\
Left-lateral and $\beta_{1}<20^{\circ}$ & Right swing and $\gamma>\beta_{1}$ \\
Left-lateral and $\beta_{1} \geq 20^{\circ}$ & Right swing and $\gamma<\beta_{1}$ \\
Straight tooth & Left swing or right swing \\
\hline
\end{tabular}

by making some mathematical conventions as follows: (1) if the tool axis swings to the left, the shaft angle $\gamma$ is stipulated to be positive, and if the tool axis swings to the right, the shaft angle $\gamma$ is negative; (2) following the direction of view shown in Figure 10, the angular velocities of the workpiece and the tool are stipulated to be positive if rotating anticlockwise or negative if rotating clockwise.

Under these conventions, the abstract motion model of skiving can be applied to different types of workpieces by the following settings:

(1) Skiving internal right-lateral tooth,

$$
\left\{\begin{array}{c}
\gamma>0, \\
\omega_{1}>0, \\
\omega_{2}>0 .
\end{array}\right.
$$

(2) Skiving internal left-lateral tooth,

$$
\left\{\begin{array}{c}
\gamma<0, \\
\omega_{1}<0, \\
\omega_{2}<0 .
\end{array}\right.
$$

(3) Skiving external right-lateral tooth,

$$
\left\{\begin{array}{c}
\gamma>0, \\
\omega_{1}>0, \\
\omega_{2}<0 .
\end{array}\right.
$$

(4) Skiving external left-lateral tooth,

$$
\left\{\begin{array}{c}
\gamma<0, \\
\omega_{1}<0, \\
\omega_{2}>0 .
\end{array}\right.
$$

(5) When skiving workpieces with straight tooth, there is no limit on the swing direction of the tool axis. For internal straight tooth, either of Case 1 or Case 2 can be adopted. For external straight tooth, either of Case 3 or Case 4 can be adopted.

4.3. Coordinate System for Skiving. According to the above motion model, a coordinate system for gear skiving is established for further mathematical derivation, as shown in Figure 11.

In Figure 11, $S_{1}\left(o_{1}, x_{1}, y_{1}, z_{1}\right)$ is the workpiece coordinate system, which is used to describe the tooth surface of the workpiece. Axis $z_{1}$ coincides with the axis of the workpiece. This coordinate system is fixed with the workpiece and 


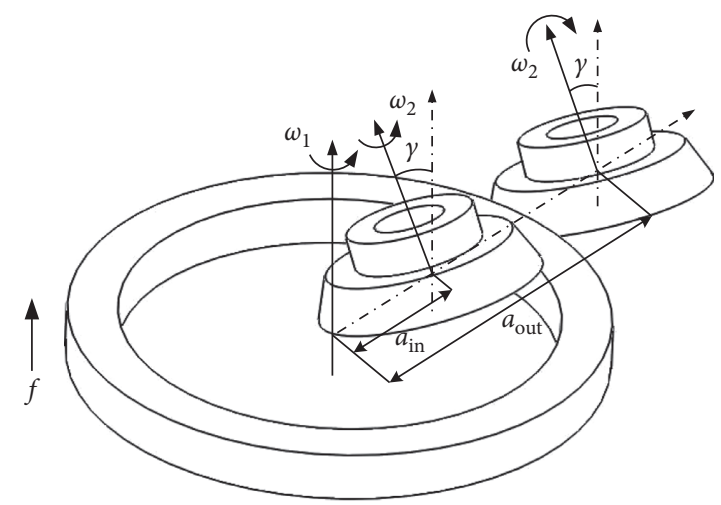

Figure 9: Motions and position relationship in skiving.

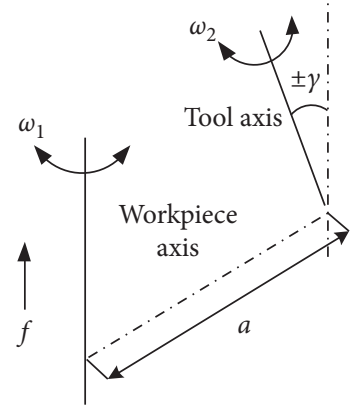

Figure 10: Abstract model of skiving.

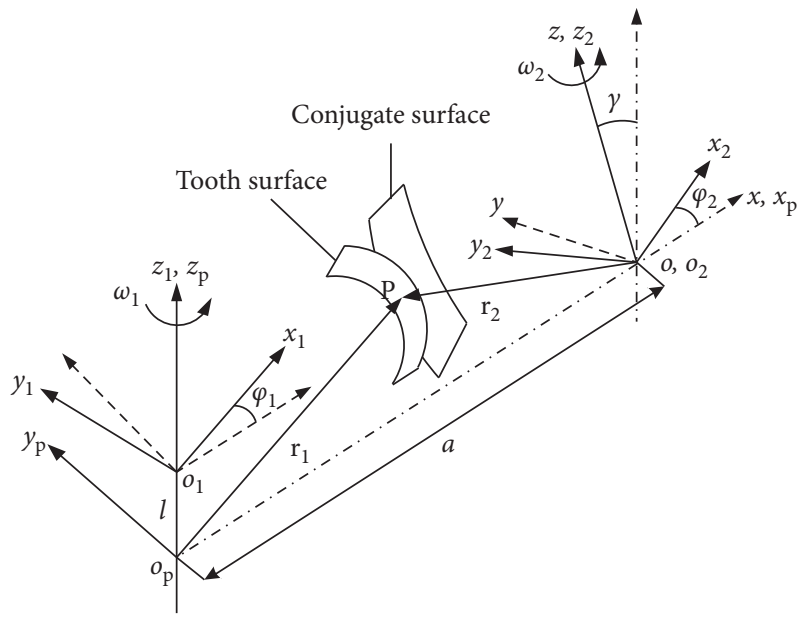

Figure 11: Coordinate systems of skiving.

moves correspondingly in the space. The coordinate system $S_{2}\left(o_{2}, x_{2}, y_{2}, z_{2}\right)$ is called tool coordinate system, which is used to establish the mathematical model of the tool. Axis $z_{2}$ coincides with the tool axis, so that the angle between axis $z_{2}$ and axis $z_{1}$ is equal to shaft angle $\gamma$, and the vertical distance between them is equal to center distance $a$. The coordinate system $S_{2}$ rotates around axis $z_{2}$ at the same speed with the tool. Specially, the moment when axis $x_{1}$ coincides with axis $x_{2}$ is regarded as the initial time. At this time, the positions of
$S_{1}$ and $S_{2}$ are represented by $S_{p}\left(o_{p}, x_{p}, y_{p}, z_{p}\right)$ and $S_{o}(o, x, y$, $z)$, respectively, which are fixed in the space. The unit vectors of axes $x_{1}, y_{1}$, and $z_{1}$ are expressed by $\mathbf{i}_{1}, \mathbf{j}_{1}$, and $\mathbf{k}_{1}$; the unit vectors of axes $x_{2}, y_{2}$, and $z_{2}$ are expressed by $\mathbf{i}_{2}, \mathbf{j}_{2}$, and $\mathbf{k}_{2}$; the unit vectors of axes $x_{\mathrm{p}}, y_{\mathrm{p}}$, and $z_{\mathrm{p}}$ are expressed by $\mathbf{i}_{p}, \mathbf{j}_{p}$, and $\mathbf{k}_{p}$; and the unit vectors of axes $x, y$, and $z$ are expressed by $\mathbf{i}, \mathbf{j}$, and $\mathbf{k}$.

\section{Mathematical Model of Conjugate Surface}

According to skiving principle, the conjugate surface is the basis of tool design. In this section, the mathematical model of conjugate surface is established according to skiving motions.

5.1. Point Contact Relation. The conjugate surface could make continuous tangent contact with the tooth surface. As shown in Figure 11, at one moment, the contact point is represented by $P$. From the initial time to this moment, the rotation angle of the workpiece is expressed by $\varphi_{1}$, and the distance of its linear movement along axis $z_{1}$ is expressed by $l$. The rotation angle of the tool at the same time is expressed by $\varphi_{2}$.

The expression of the tooth surface is given in the workpiece coordinate system $S_{1}$ before tool design and briefly expressed as

$$
\mathbf{r}_{1}=\mathbf{r}_{1}(\theta, \mu)+x_{1}(\theta, \mu) \mathbf{i}_{1}+y_{1}(\theta, \mu) \mathbf{j}_{1}+z_{1}(\theta, \mu) \mathbf{k}_{1},
$$

where $\theta$ and $\mu$ are variables of the tooth surface. 
In the tool coordinate system $S_{2}$, the conjugate surface is assumed as

$$
\mathbf{r}_{2}=x_{2} \mathbf{i}_{2}+y_{2} \mathbf{j}_{2}+z_{2} \mathbf{k}_{2}
$$

It is noticed that the point $P$ on the tooth surface coincides with that on the conjugate surface at this moment. Therefore, their coordinates are the same in one coordinate system. The coordinates of the point on the tooth surface are transformed from $S_{1}$ to $S_{2}$ as follows:

$$
\left[\begin{array}{c}
x_{2} \\
y_{2} \\
z_{2} \\
1
\end{array}\right]=M_{21}\left[\begin{array}{l}
x_{1} \\
y_{1} \\
z_{1} \\
1
\end{array}\right],
$$

where $M_{21}$ means the transformation matrix. With the help of $S_{o}$ and $S_{p}$, we get

$$
M_{21}=M_{20} M_{0 p} M_{p 1} .
$$

$$
\begin{aligned}
M_{20} & =\left[\begin{array}{cccc}
\cos \varphi_{2} & \sin \varphi_{2} & 0 & 0 \\
-\sin \varphi_{2} & \cos \varphi_{2} & 0 & 0 \\
0 & 0 & 1 & 0 \\
0 & 0 & 0 & 1
\end{array}\right], \\
M_{0 p} & =\left[\begin{array}{cccc}
1 & 0 & 0 & a \\
0 & \cos \gamma & -\sin \gamma & 0 \\
0 & \sin \gamma & \cos \gamma & 0 \\
0 & 0 & 0 & 1
\end{array}\right], \\
M_{p 1} & =\left[\begin{array}{cccc}
\cos \varphi_{1} & -\sin \varphi_{1} & 0 & 0 \\
\sin \varphi_{1} & \cos \varphi_{1} & 0 & 0 \\
0 & 0 & 1 & -l \\
0 & 0 & 0 & 1
\end{array}\right] .
\end{aligned}
$$

By equations (5)-(8), the expression of the conjugate surface can be obtained as

According to Figure 11, the intermediate transformation matrixes are obtained as follows:

$$
\left\{\begin{aligned}
x_{2}= & x_{1}(\theta, \mu)\left(\cos \varphi_{1} \cos \varphi_{2}+\sin \varphi_{1} \sin \varphi_{2} \cos \gamma\right)+y_{1}(\theta, \mu)\left(-\sin \varphi_{1} \cos \varphi_{2}+\cos \varphi_{1} \sin \varphi_{2} \cos \gamma\right) \\
& -z_{1}(\theta, \mu) \sin \varphi_{2} \sin \gamma+l \sin \varphi_{2} \sin \gamma+a \cos \varphi_{2}, \\
y_{2}= & x_{1}(\theta, \mu)\left(-\cos \varphi_{1} \sin \varphi_{2}+\sin \varphi_{1} \cos \varphi_{2} \cos \gamma\right)+y_{1}(\theta, \mu)\left(\sin \varphi_{1} \sin \varphi_{2}+\cos \varphi_{1} \cos \varphi_{2} \cos \gamma\right) \\
& -z_{1}(\theta, \mu) \cos \varphi_{2} \sin \gamma+l \cos \varphi_{2} \sin \gamma-a \sin \varphi_{2} \\
z_{2}= & x_{1}(\theta, \mu) \sin \varphi_{1} \sin \gamma+y_{1}(\theta, \mu) \cos \varphi_{1} \sin \gamma+z_{1}(\theta, \mu) \cos \gamma-l \cos \gamma
\end{aligned}\right.
$$

where $\varphi_{1}, \varphi_{2}$, and $l$ are unknown. They satisfy the following relations:

$$
\begin{aligned}
l & =f \frac{\varphi_{1}}{\omega_{1}}, \\
\varphi_{2} & =\varphi_{2} \frac{\omega_{2}}{\omega_{1}} .
\end{aligned}
$$

Therefore, there is only one independent variable in equation (10). This variable can be solved according to the conjugate relation.

5.2. Conjugate Relation. According to the theory of conjugate surface, the common normal vector $\mathbf{n}$ of the tooth surface and the conjugate surface at point $P$ is perpendicular to the relative velocity $\mathbf{v}_{21}$. So,

$$
\mathbf{n} \cdot \mathbf{v}_{21}=0 \text {. }
$$

The premise of this equation is that $\mathbf{n}$ and $\mathbf{v}_{21}$ are expressed in the same coordinate system. The expression of $\mathbf{n}$ in the coordinate system $S_{1}$ can be derived from equation (5) as

$$
\begin{aligned}
\mathbf{n} & =\frac{\partial \mathbf{r}_{1}}{\partial \theta} \times \frac{\partial \mathbf{r}_{1}}{\partial \mu}=\left|\begin{array}{ccc}
\mathbf{i}_{1} & \mathbf{j}_{1} & \mathbf{k}_{1} \\
\frac{\partial x_{1}}{\partial \theta} & \frac{\partial y_{1}}{\partial \theta} & \frac{\partial x_{1}}{\partial \theta} \\
\frac{\partial x_{1}}{\partial \mu} & \frac{\partial y_{1}}{\partial \mu} & \frac{\partial z_{1}}{\partial \mu}
\end{array}\right| \\
& =n_{x} \mathbf{i}_{1}+n_{y} \mathbf{j}_{1}+n_{z} \mathbf{k}_{1},
\end{aligned}
$$

where $n_{x}, n_{y}$, and $n_{z}$ are, respectively, the components of $\mathbf{n}$ on axes $x_{1}, y_{1}$, and $z_{1}$ : 


$$
\left\{\begin{array}{l}
n_{x}=\left|\begin{array}{ll}
\frac{\partial y_{1}}{\partial \theta} & \frac{\partial z_{1}}{\partial \theta} \\
\frac{\partial y_{1}}{\partial \mu} & \frac{\partial z_{1}}{\partial \mu}
\end{array}\right|=\frac{\partial y_{1}}{\partial \theta} \frac{\partial z_{1}}{\partial \mu}-\frac{\partial y_{1}}{\partial \mu} \frac{\partial z_{1}}{\partial \theta} \\
n_{y}=\left|\begin{array}{ll}
\frac{\partial z_{1}}{\partial \theta} & \frac{\partial x_{1}}{\partial \theta} \\
\frac{\partial z_{1}}{\partial \mu} & \frac{\partial x_{1}}{\partial \mu}
\end{array}\right|=\frac{\partial z_{1}}{\partial \theta} \frac{\partial x_{1}}{\partial \mu}-\frac{\partial z_{1}}{\partial \mu} \frac{\partial x_{1}}{\partial \theta} \\
n_{z}=\left|\begin{array}{ll}
\frac{\partial x_{1}}{\partial \theta} & \frac{\partial y_{1}}{\partial \theta} \\
\frac{\partial x_{1}}{\partial \mu} & \frac{\partial y_{1}}{\partial \mu}
\end{array}\right|=\frac{\partial x_{1}}{\partial \theta} \frac{\partial y_{1}}{\partial \mu}-\frac{\partial x_{1}}{\partial \mu} \frac{\partial y_{1}}{\partial \theta}
\end{array}\right.
$$

As shown in Figure 11, at point $P$, the relative velocity can be calculated by

$$
\mathbf{v}_{21}=\boldsymbol{\omega}_{2} \times \mathbf{r}_{2}-\boldsymbol{\omega}_{1} \times \mathbf{r}_{1}-\mathbf{f} .
$$

Among them,

$$
\left\{\begin{aligned}
\boldsymbol{\omega}_{1} & =\omega_{1} \mathbf{k}_{1}, \\
\boldsymbol{\omega}_{2} & =\omega_{2} \mathbf{k}_{2}, \\
\mathbf{v} & =f \mathbf{k}_{1}, \\
\mathbf{r}_{1} & =x_{1} \mathbf{i}+y_{1} \mathbf{j}+z_{1} \mathbf{k}_{1}, \\
\mathbf{r}_{2} & =\overrightarrow{O O_{\mathrm{p}}}+\mathbf{r}_{1}-\left(-a \mathbf{i}_{p}\right)+x_{1} \mathbf{i}_{1}+y_{1} \mathbf{j}_{1}+z_{1} \mathbf{k}_{1} .
\end{aligned}\right.
$$

The unit vectors of coordinate axes in equation (17) satisfy the following equations:

$$
\begin{aligned}
& {\left[\begin{array}{c}
\mathbf{i}_{2} \\
\mathbf{j}_{2} \\
\mathbf{k}_{2} \\
1
\end{array}\right]=M_{21}^{n}\left[\begin{array}{c}
\mathbf{i}_{1} \\
\mathbf{j}_{1} \\
\mathbf{k}_{1} \\
1
\end{array}\right],} \\
& {\left[\begin{array}{c}
\mathbf{i}_{p} \\
\mathbf{j}_{p} \\
\mathbf{k}_{p} \\
1
\end{array}\right]=M_{\mathrm{p} 1}^{n}\left[\begin{array}{c}
\mathbf{i}_{1} \\
\mathbf{j}_{1} \\
\mathbf{k}_{1} \\
1
\end{array}\right],}
\end{aligned}
$$

where

$$
M_{21}^{n}=M_{20}^{n} M_{0 p}^{n} M_{p 1}^{n} .
$$

The intermediate matrixes in equation (20) for vector transformation can be obtained by setting the part, which is related to the displacement of coordinate origin, of the matrixes in equation (8) to be 0 .

By simplifying equations (18)-(20), we get

$$
\left\{\begin{array}{l}
\mathbf{k}_{2}=\mathbf{i}_{1} \sin \varphi_{1} \sin \gamma+\mathbf{j}_{1} \cos \varphi_{1} \sin \gamma+\mathbf{k}_{1} \cos \gamma \\
\mathbf{i}_{p}=\mathbf{i}_{1} \cos \varphi_{1}-\mathbf{j}_{1} \sin \varphi_{1} .
\end{array}\right.
$$

Equations (17) and (21) are introduced into equation (16), and then the relative velocity can be obtained as follows:

$$
\begin{aligned}
& \mathbf{v}_{21}=v_{x} \mathbf{i}_{1}+v_{y} \mathbf{j}_{1}+v_{z} \mathbf{k}_{1}, \\
& \left\{\begin{array}{l}
v_{x}=z_{z} \omega_{2} \cos \varphi_{1} \sin \gamma-\omega_{2}\left(y_{1}+a \sin \varphi_{1}\right) \cos \gamma+y_{1} \omega_{1}, \\
v_{y}=\left(x_{1}-a \cos \varphi_{1}\right) \omega_{2} \cos \gamma-z_{1} \omega_{2} \sin \varphi_{1} \sin \gamma-x_{1} \omega_{1}, \\
v_{z}=y_{1} \omega_{2} \sin \varphi_{1} \sin \gamma-x_{1} \omega_{2} \cos \varphi_{1} \sin \gamma-f .
\end{array}\right.
\end{aligned}
$$

According to equation (13), the following equation can be obtained:

$$
n_{x} v_{x}+n_{y} v_{y}+n_{z} v_{z}=0 .
$$

By introducing equations (15) and (23) into equation (24), the following equations are obtained:

$$
U \sin \varphi_{1}-V \cos \varphi_{1}=W,
$$

$$
\left\{\begin{array}{l}
U=-n_{x} a \omega_{2} \cos \gamma-n_{y} z_{1} \omega_{2} \sin \gamma+n_{z} y_{1} \omega_{2} \sin \gamma \\
V=-n_{x} z_{1} \omega_{2} \sin \gamma+n_{y} a \omega_{2} \cos \gamma+n_{z} x_{1} \omega_{2} \sin \gamma \\
W=\left(n_{x} y_{1}-n_{y} x_{1}\right)\left(\omega_{2} \cos \gamma-\omega_{1}\right)+n_{z} f
\end{array}\right.
$$

5.3. Analytic Expression of the Conjugate Surface. Solved by equations (25) and (26), the unknown quantity $\varphi_{1}$ can be obtained:

$$
\varphi_{1}=\arcsin \left(\frac{U W \pm V \sqrt{U^{2}+V^{2}-W^{2}}}{U^{2}+V^{2}}\right) .
$$

In order to ensure that $\varphi_{1}$ is an effective real number, the following requirement should be satisfied:

$$
U^{2}+V^{2}-W^{2} \geq 0 .
$$

There are two possible solutions for $\varphi_{1}$ as shown in equation (27). When applying equation (27) in practice, one appropriate solution should be selected according to the condition that whether the absolute value of the part in the bracket is less than 1 .

The analytic expression of the conjugate surface is then obtained by introducing equations (11), (12), and (27) into equation (10). It can be seen that the conjugate surface shows as a space surface with parameters $\theta$ and $\mu$, which are taken from the tooth surface. Due to the space limitation of this article, the conjugate surface is expressed briefly as

$$
\mathbf{r}_{2}=\mathbf{r}_{2}(\theta, \mu)=x_{2}(\theta, \mu) \mathbf{i}_{2}+y_{2}(\theta, \mu) \mathbf{j}_{2}+z_{2}(\theta, \mu) \mathbf{k}_{2} .
$$

5.4. Periodicity of the Conjugate Relation. The common cylindrical gear is generally composed of several evenly distributed teeth, and all the teeth can be obtained by circular 
array of one tooth. If the number of teeth is $Z_{1}$, then the angle between two adjacent teeth is

$$
\psi_{1}=\frac{2 \pi}{Z_{1}}
$$

Similarly, the tool also consists of several evenly distributed teeth. If the number of tool teeth is $Z_{2}$, then the angle between two adjacent teeth is

$$
\psi_{2}=\frac{2 \pi}{Z_{2}}
$$

It is assumed that two adjacent teeth $T_{1}$ and $T_{2}$ of the workpiece would be cut by tool teeth $D_{1}$ and $D_{2}$, respectively. The conjugate surface, where the cutting edge of $D_{1}$ is located, is calculated by the method in Sections 4.1-4.3. In the workpiece coordinate system, $T_{2}$ is obtained from $T_{1}$ by circular array according to $\psi_{1}$. In the tool coordinate system, $D_{2}$ is obtained from $D_{1}$ by circular array according to $\psi_{2}$.

When there is no axial feed, if the workpiece rotates around its own axis for angle $\psi_{1}$, the tooth $T_{2}$ would come to the position where $T_{1}$ was located before rotation. At the same time, if the tool rotates around its own axis just for $\psi_{2}$, the tooth $D_{2}$ would come to the position where $D_{1}$ was located before rotation. The conjugate relation between $T_{1}$ and $D_{1}$ would be repeated by $T_{2}$ and $D_{2}$. In order to satisfy this condition, we must have

$$
\frac{\psi_{1}}{\psi_{2}}=\frac{\left|\omega_{1}\right|}{\left|\omega_{2}\right|}=\frac{Z_{2}}{Z_{1}} .
$$

Therefore, in gear skiving, the tool and the workpiece must rotate synchronously under the inverse ratio of the number of teeth. Only in this way, the conjugate relation is periodic, and just one tooth is needed to be calculated in the process of tool design.

In fact, there is axial feed movement in gear skiving. For helical gear, an additional rotation of the workpiece must be adopted to maintain the periodicity of the conjugate relation. The angular velocity of the additional rotation of the workpiece is related to the axial feed rate $f$. It is assumed as

$$
\Delta \omega_{1}=i_{f} f .
$$

According to equations (25), (26), and (33), the conjugate relation that contains additional rotation changes to be

$$
\begin{gathered}
U_{f} \sin \varphi_{1}^{f}-V_{f} \cos \varphi_{1}^{f}=W_{f}, \\
V_{f}=-n_{x} z_{1} \omega_{2} \sin \gamma+n_{y} a \omega_{2} \cos \gamma+n_{z} x_{1} \omega_{2} \sin \gamma, \\
W_{f}=\left(n_{x} y_{1}-n_{y} x_{1}\right)\left[\omega_{2} \cos \gamma-\left(\frac{\omega_{2} Z_{2}}{Z_{1}+i_{f} f}\right)\right]+n_{z} f,
\end{gathered}
$$

that is,

$$
\begin{aligned}
& {\left[\left(-n_{x} a \cos \gamma-n_{y} z_{1} \sin \gamma+n_{z} y_{1} \sin \gamma\right) \sin \varphi_{1}^{f}-\left(-n_{x} z_{1} \sin \gamma+n_{y} a \cos \gamma+n_{z} x_{1} \sin \gamma\right) \cos \varphi_{1}^{f}\right]} \\
& \omega_{2}+i_{f}\left(n_{x} y_{1}-n_{y} x_{1}\right) f=\left(n_{x} y_{1}-n_{y} x_{1}\right)\left(\cos \gamma-\frac{Z_{2}}{Z_{1}}\right) \omega_{2}+n_{z} f .
\end{aligned}
$$

In equation (36), $\omega_{2}$ and $f$ are independent parameters. In order to make the equation true, we must make the factors of $\omega_{2}$ and $f$ be the same, respectively. Then, we can get

$$
i_{f}\left(n_{x} y_{1}-n_{y} x_{1}\right)=n_{z}
$$

that is,

$$
\Delta \omega_{1}=\frac{n_{z}}{n_{x} y_{1}-n_{y} x_{1}} f .
$$

It can be seen from equation (38) that the angular speed of the additional rotation is related to the feed rate $f$ and the shape of the tooth surface. There are several special cases as follows:

(1) For straight tooth, $n_{z}=0$. Therefore, no additional rotation is needed.
(2) For helical involute gear, by putting the equation of involute spiral surface into equation (38), it can be obtained:

$$
\Delta \omega_{1}=\frac{1}{p} f
$$

where $p$ is the spiral parameter of the tooth surface. Since $p$ is a constant value, the additional rotation would remain constant in the cutting process.

(3) For noninvolute helical gear, generally speaking, the additional rotation cannot keep constant so that gear skiving is inapplicable.

To sum up, involute gear and straight noninvolute gear can be manufactured by skiving technology. The angular velocity of the additional rotation is also determined by the rotation direction of the workpiece. Taking the cases in 
Section 4.2 into consideration, the more specific parameter settings regarding additional rotation are listed as follows:

(1) Skiving internal right-lateral tooth,

$$
\left\{\begin{array}{l}
\gamma>0, \\
\omega_{2}>0, \\
\omega_{1}=\frac{\omega_{2} Z_{2}}{Z_{1}+i_{\mathrm{f}} f} .
\end{array}\right.
$$

(2) Skiving internal left-lateral tooth,

$$
\left\{\begin{array}{l}
\gamma<0 \\
\omega_{2}<0 \\
\omega_{1}=\frac{\omega_{2} Z_{2}}{Z_{1}-i_{\mathrm{f}} f}
\end{array}\right.
$$

(3) Skiving external right-lateral tooth,

$$
\left\{\begin{array}{l}
\gamma>0, \\
\omega_{2}<0, \\
\omega_{1}=\frac{\omega_{2} Z_{2}}{Z_{1}+i_{f} f} .
\end{array}\right.
$$

(4) Skiving external left-lateral tooth,

$$
\left\{\begin{array}{l}
\gamma<0, \\
\omega_{2}>0, \\
\omega_{1}=\frac{\omega_{2} Z_{2}}{Z_{1}-i_{\mathrm{f}} f} .
\end{array}\right.
$$

(5) Skiving internal straight noninvolute tooth,

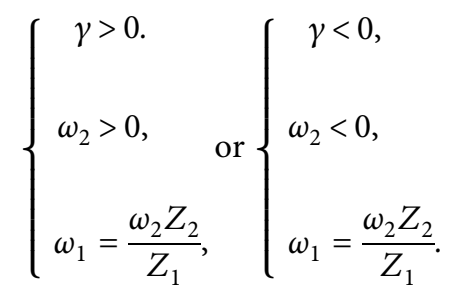

(6) Skiving external straight noninvolute tooth,

$$
\left\{\begin{array} { l } 
{ \gamma > 0 , } \\
{ \omega _ { 2 } < 0 , } \\
{ \omega _ { 1 } = \frac { - \omega _ { 2 } Z _ { 2 } } { Z _ { 1 } } , }
\end{array} \text { or } \left\{\begin{array}{l}
\gamma<0, \\
\omega_{2}>0, \\
\omega_{1}=\frac{-\omega_{2} Z_{2}}{Z_{1}} .
\end{array}\right.\right.
$$

\section{Tool Design Example}

In order to verify the feasibility of the proposed method, this section provides an example aiming at internal involute gear. The parameters of the workpiece are listed in Table 2. The tool parameters and processing parameters are set according to the workpiece parameters, as listed in Tables 3 and 4.

Based on the workpiece parameters, the mathematical model of the tooth surface is established as

$$
\left\{\begin{array}{l}
x_{1}^{i}=r_{b} \cos \left(\sigma_{0}+\mu+\theta\right)+r_{b} \mu \sin \left(\sigma_{0}+\mu+\theta\right), \\
x_{1}^{i}=r_{b} \sin \left(\sigma_{0}+\mu+\theta\right)+r_{b} \mu \cos \left(\sigma_{0}+\mu+\theta\right), \\
z_{1}^{i}=p \theta
\end{array}\right.
$$

where $\mu$ and $\theta$ are variables of this equation.

Then, the normal vector of the tooth surface is calculated by

$$
\left\{\begin{array}{l}
n_{x i}-p r_{b} \mu \sin \left(\sigma_{0}+\mu+\theta\right) \\
n_{y i}-p r_{b} \mu \cos \left(\sigma_{0}+\mu+\theta\right) \\
n_{z i}=\mu r_{b}^{2}
\end{array}\right.
$$

Since the workpiece is internal and right-lateral, the shaft angle and the angular velocities are set as follows:

$$
\left\{\begin{aligned}
\gamma & =20^{\circ} \\
\omega_{2} & =\frac{2 \pi n_{2}}{60}=157 \\
\omega_{1} & =\frac{\omega_{2} Z_{2}}{Z_{1}+i_{\mathrm{f}} f}=\frac{\omega_{2} Z_{2}}{Z_{1}}+\frac{f}{p}=54.186 .
\end{aligned}\right.
$$

6.1. Design of Cutting Edge. Based on the above settings, the mathematical model of the conjugate surface is established. For simplicity, the cutting edge is designed to be the intersecting curve between the conjugate surface and an auxiliary plane as shown in Figure 12. This plane is constructed according to the helix angle $\beta_{2}$ of the tool and a shape-rake-angle $\gamma_{\mathrm{e}}$ which should be given before tool design. To be specific, a vector is constructed along the spiral direction of the tool tooth, expressed by $\mathbf{l}_{b}$. The projection of point $D$ on axis $z_{2}$ is found and expressed by $D z$. In the plane that determined by $\mathbf{l}_{b}$ and line $D D_{z}$, a vector (expressed by $\mathbf{n}_{p}$ ) is constructed, which has an angle of $\gamma_{e}$ with $\mathbf{1}_{b}$. Taking $\mathbf{n}_{p}$ as the normal vector, a plane is constructed as the abovementioned auxiliary plane. Then, the cutting edge is obtained by calculating the intersecting curve between the conjugate surface and the auxiliary plane. In this example, the angle $\gamma_{e}$ is set to be $5^{\circ}$. Due to the limitation of article length, the specific calculation process is not presented.

6.2. Design of Rake Face. According to the design principle proposed in Section 3, the rake face is constructed on the basis of the cutting edge. In this example, the rake face is 
TABLE 2: Basic parameters of the involute gear.

\begin{tabular}{lccccccc}
\hline $\begin{array}{l}\text { Teeth } \\
\text { number }\end{array}$ & $\begin{array}{c}\text { Normal } \\
\text { module }(\mathrm{mm})\end{array}$ & $\begin{array}{c}\text { Tooth profile } \\
\text { angle }\end{array}$ & $\begin{array}{c}\text { Helix } \\
\text { angle }\end{array}$ & $\begin{array}{c}\text { Addendum circle } \\
\text { diameter }(\mathrm{mm})\end{array}$ & $\begin{array}{c}\text { Dedendum circle } \\
\text { diameter }(\mathrm{mm})\end{array}$ & $\begin{array}{c}\text { Modification } \\
\text { coefficient }\end{array}$ & $\begin{array}{c}\text { Tooth width } \\
(\mathrm{mm})\end{array}$ \\
\hline 113 & 1.9 & $17.5^{\circ}$ & $20^{\circ}$ & 226.281 & 235.781 & 0.5214 & 54 \\
\hline
\end{tabular}

TABLE 3: Skiving tool parameters.

\begin{tabular}{lcc}
\hline Teeth number $Z_{2}$ & Working rake angle $\gamma_{o}$ & Relief angle $\alpha_{d}$ \\
\hline 39 & $10^{\circ}$ & $6.5^{\circ}$ \\
\hline
\end{tabular}

TABLE 4: Machining parameters.

\begin{tabular}{lcc}
\hline Center distance $(\mathrm{mm})$ & Axial feed rate & Tool rotation speed \\
\hline 78.3 & $0.1 \mathrm{~mm} / \mathrm{r}$ & $1500 \mathrm{r} / \mathrm{min}$ \\
\hline
\end{tabular}

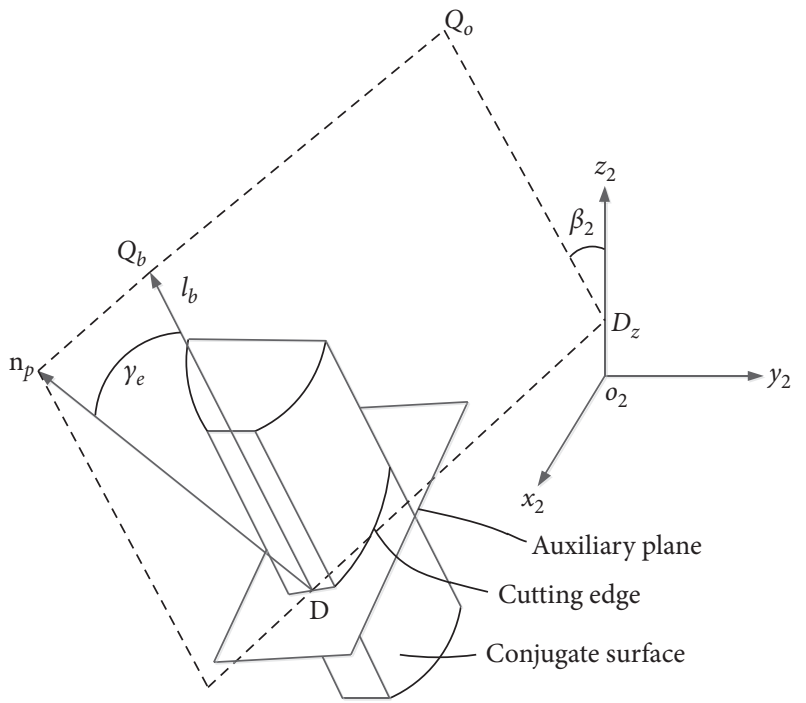

Figure 12: Schematic diagram of the construction method of the cutting edge.

designed to be a curved surface to achieve required working rake angle. As shown in Figure 13, a coordinate system for cutting angles is established at point $D$ of the cutting edge according to the cutting velocity $v_{21}$ and the tangent vector $\boldsymbol{t}_{r}$ of the cutting edge. In this coordinate system, $P_{s}$ means the cutting plane which passes through $v_{21}$ and $\boldsymbol{t}_{\mathrm{r}} . P_{r}$ means the base plane which is perpendicular to $v_{21}$. And $P_{o}$ means the orthogonal plane which is perpendicular to both $P_{s}$ and $P_{r}$.

According to the metal cutting theory, the working rake angle shows as the included angle between two straight lines in the orthogonal plane $P_{o}$. One of them is the tangent line of the intersecting curve between the rake face and $P_{o}$, and the other one is the intersecting line between $P_{o}$ and $P_{r} . \mathbf{N}_{o}$ and $N_{1}$ represent the unit direction vectors of these two lines, respectively. Since $N_{1}$ is perpendicular to plane $P_{s}$, we can obtain

$$
\mathbf{N}_{1}=\frac{\mathbf{v}_{21} \times \mathbf{t}_{r}}{\left|\mathbf{v}_{21} \times \mathbf{t}_{r}\right|}
$$

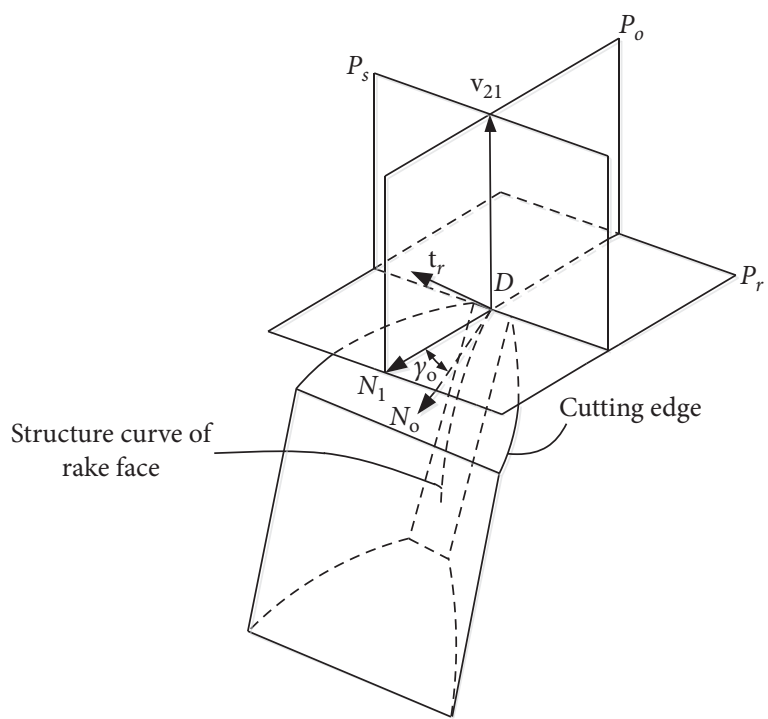

FIGURE 13: Schematic diagram of the construction principle of the rake face.

In the orthogonal plane $P_{o}$, with a given working rakeangle $\gamma_{o}$, vector $\mathbf{N}_{o}$ can be obtained by

$$
\mathbf{N}_{o}=\frac{-\sin \left(\gamma_{o}\right) \mathbf{v}_{21}+\cos \left(\gamma_{o}\right) \mathbf{N}_{1}}{\left|-\sin \left(\gamma_{o}\right) \mathbf{v}_{21}+\cos \left(\gamma_{o}\right) \mathbf{N}_{1}\right|}
$$

This vector shows as the tangent vector of the rake face at point $D$. Here, it is used in reverse to construct the rake face. Specifically, a parabola curve is calculated by taking $\mathbf{N}_{o}$ as its tangent vector and making itself pass through point $D$. Similarly, several curves can be constructed at several discrete points of the cutting edge. And then, the rake face is constructed by interpolating the points on these curves using cubic B-spline surface:

$$
\mathbf{q}_{i}(u, w)=\sum_{i=0}^{E-1} \sum_{j=0}^{F-1} \mathbf{P}_{i, j} N_{i, 3}(u) N_{i, 3}(w), \quad 0 \leq u, w \leq 1 .
$$

6.3. Design of Flank Face. In order to ensure the shape precision of the cutting edge that would be obtained after tool regrinding, the flank face is constructed by several ideal cutting edges $e_{1}, e_{2}, \ldots$, as shown in Figure 14. These curves also show as the intersection of conjugate surface and auxiliary plane. In order to form relief angle, when calculating the conjugate surface corresponding to every time of tool regrinding, the center distance changes for $\Delta g$, and the auxiliary plane moves parallelly for distance $\Delta d$. The ratio of them depends on the given relief angle $\alpha_{d}$, that is, 


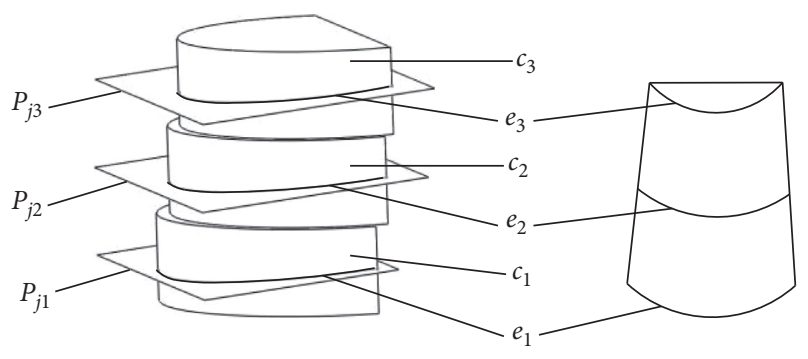

FIGURE 14: Schematic diagram of the construction principle of the flank face.

$$
\alpha_{d}=\arccos \left(\frac{\Delta d}{\Delta g}\right) .
$$

In this example, the distance $\Delta d$ is set to be $0.5 \mathrm{~mm}$, and then plane $P_{j 2}$ can be obtained on the basis of $P_{j 1}$ which is constructed in Section 6.1. The number of times that the tool could be reground is set to be 20 . So, the effective length of the flank face is $20 \times \Delta d=10 \mathrm{~mm}$. Then, $\Delta \mathrm{g}$ is calculated by equation (52), and conjugate surface $c_{2}$ is constructed by the method proposed in Section 5. On this basis, cutting edge $e_{2}$ can be obtained by calculating the intersecting curve of conjugate surface $c_{2}$ and plane $P_{j 2}$. By analogy, all the 20 cutting edges can be obtained, and the flank face is constructed by interpolating the points on these curves using $\mathrm{B}$-spline surface:

$$
\mathbf{h}_{i}(u, w)=\sum_{i=0}^{H-1} \sum_{j=0}^{I-1} \mathbf{Q}_{i, j} N_{i, 3}(u) N_{i, 3}(w), \quad 0 \leq u, w \leq 1 .
$$

\subsection{Theoretical Derivations of Interference and Undercutting.} The previous research indicated that interference [6] and undercutting may occur if skiving parameters were not properly selected, which not only affected the processing quality but also aggravated the wear of the cutter. It is necessary to do some theoretical derivations about the interference and undercutting. For this, the machined tooth surface should be derived, and the position relation of the tool surface and the gear surface should be investigated. Here, edge-sweeping surfaces are used.

In the process of skiving, the moving track of the cutting edge is named as edge-sweeping surface. The gear surface is theoretically shaped by multiple edge-sweeping surfaces as shown in Figure 15. The cluster of edge-sweeping surfaces can be derived using the mathematical model of the designed cutting edge in Section 6.1 and the transformation matrixes in Section 5.1 by the method proposed in reference [7]. It can be seen that the cutting edge generates a tangent trace line on the tooth surface with continuous sweeping. Since the conjugate relationship is periodic, every edge-sweeping surface has a contact line with the tooth surface. Due to axial

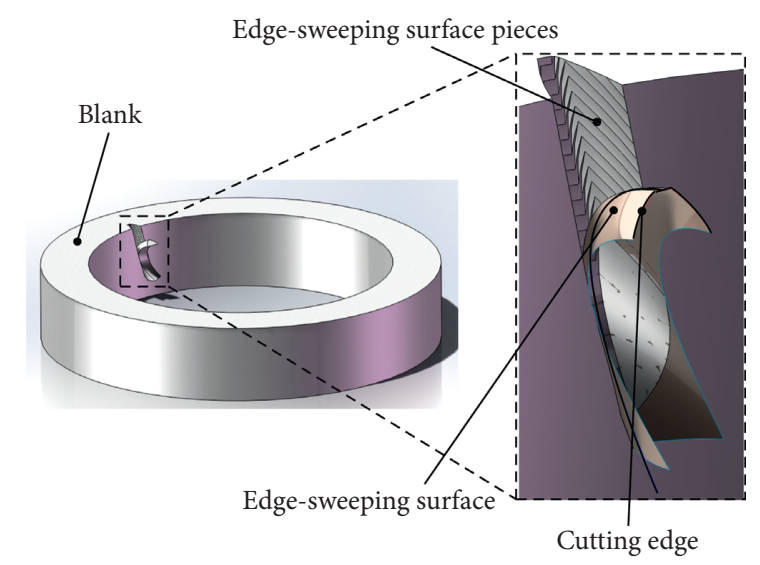

FIGURE 15: Schematic diagram of tooth surface formation.

feed, the newly formed edge-sweeping surface moves for a certain distance along the tooth direction of the workpiece. The tooth surface is formed by the fragments left after intersecting with each other of several edge-sweeping surfaces. Obviously, the intersecting curve of two adjacent edgesweeping surfaces is most prone to interference as shown in Figure 16. Therefore, it is regarded as the interference judgment criterion whether the intersecting curve of edgesweeping surfaces intersects with the flank face. In this paper, the secant method is used to solve the intersecting curves. The solving process will not be repeated here due to space limitation. In this way, the gear surface in this example can be obtained, from which interference and undercutting can be judged.

6.5. Cutting Simulation. According to the calculation results, the 3D model of the tool is built as shown in Figure 17. It can be seen that the tool faces are smooth with obvious cutting angles. The tool tooth is straight, which is consistent with the analysis in Section 4.2.

In order to verify the correctness of the proposed tool design method, a cutting simulation has been done. According to the motion model of gear skiving, the simulation model (see Figure 18) was built using the 3D models of the designed tool and the workpiece blank. As shown in Figure 19, the simulated tooth surface was smooth without overcut or collision, which indicated that no interference occurred in the cutting process. This result proved that the designed flank face was reasonable.

The simulated tooth surface was further compared with the theoretical tooth surface to investigate the shape error as shown in Figure 20. Since this process is geometric, the accuracy of the 3D model would affect the simulation precision obviously. In this example, the mode "extreme precision" was employed, and the number of triangular patches of the workpiece model in format STL reached more than 10,000 . On this condition, it was found that the processed tooth surface in green fitted well with the theoretical 


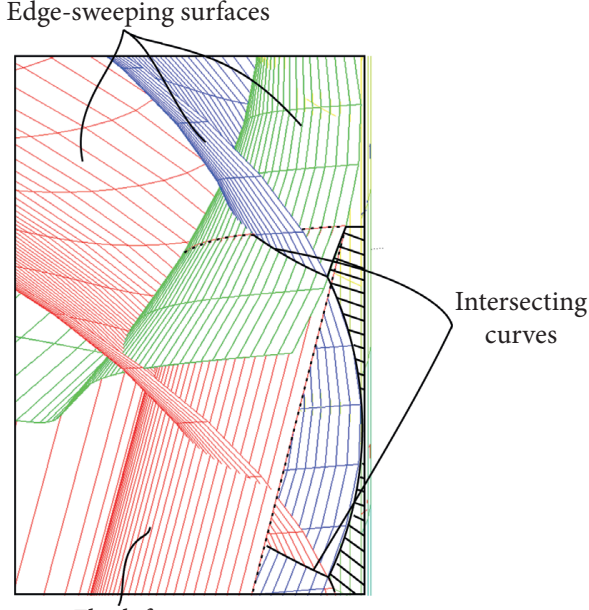

Flank face

FIgURE 16: Schematic diagram of interference.

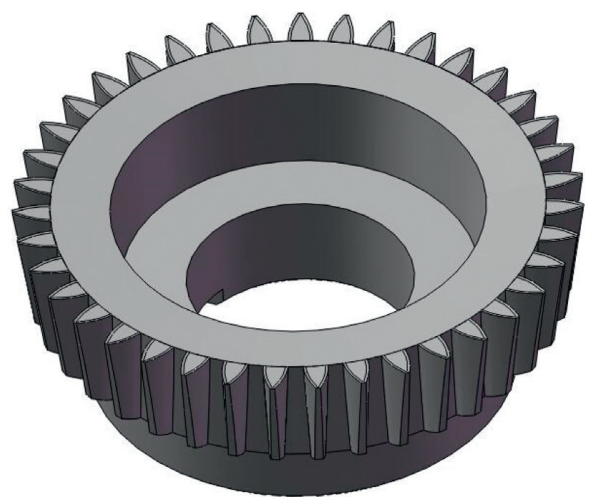

FIgUre 17: 3D model of the involute skiving tool.

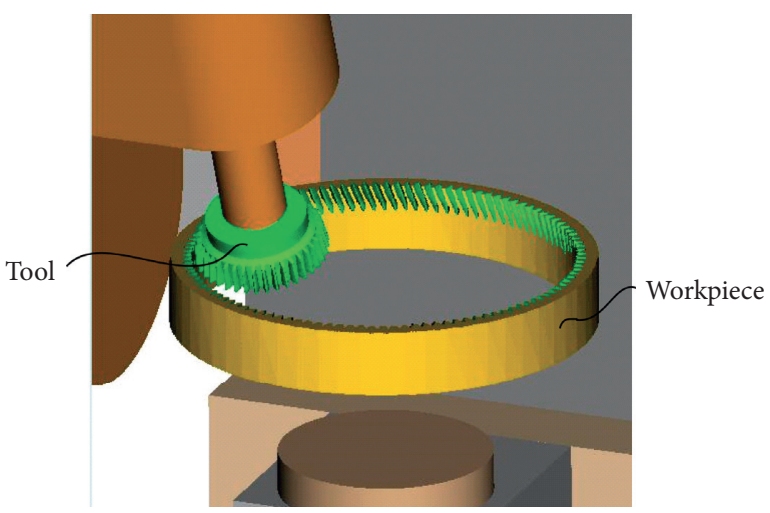

FIgURE 18: Cutting simulation model.

tooth surface in purple. The deviation of tooth profile was automatically measured by the simulation software at about $0.005 \mathrm{~mm}$. This result confirmed that the proposed design method of the cutting edge is correct.

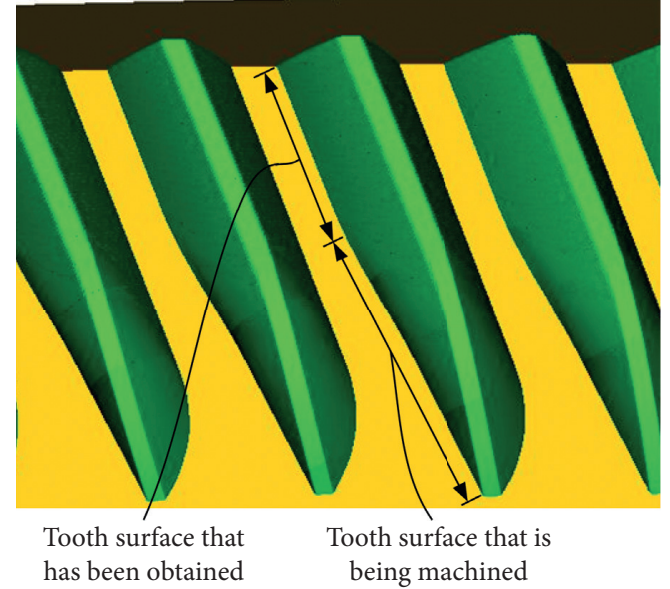

Figure 19: The simulated tooth surface.

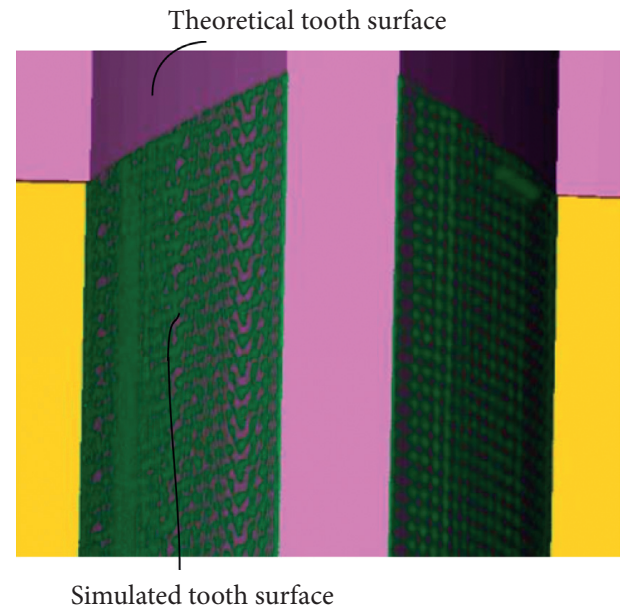

Figure 20: Comparison of tooth surface.

\section{Conclusions}

In this paper, the skiving principle is investigated firstly from the view of the essence of skiving motions. Then, the tool design principle is analyzed based on the theory of conjugate surface, and a new tool design method is proposed to match the skiving principle. Before tool design, the skiving patterns for various kinds of workpieces are enumerated and summarized to abstract a normalized motion model, and then a skiving coordinate system is accordingly established. The mathematical model of the conjugate surface is then derived based on the normalized motion model. An example is presented, and a simulation is done at last. From these research studies, the following conclusions can be drawn:

(1) The tooth profile is formed by the conjugate rotary cutting of the cutting edge under skiving motions. This principle makes it possible to shape the tooth profile integrally and directly by the main cutting 
motions, rather than enveloping. Therefore, the gear skiving is theoretically inferred to have higher profile accuracy than traditional enveloping methods.

(2) The proposed tool design method has better applicability and flexibility. The cutting edge is designed firstly by intersecting the conjugate surface with an auxiliary plane. This method is applicable to not only common involute gear but also straight noninvolute gear since the conjugate relation between the cutting edge and the tooth surface can keep periodic under a constant additional rotation. The tool faces are constructed on the basis of the cutting edge. They could be free-form curved surfaces shaped by the working angles or other factors to improve the cutting performance of the skiving tool.

(3) The proposed method was applied to the tool design for an involute gear as an example. The simulation results proved that the proposed tool design method is correct and valid. This research lays a foundation for further application in engineering.

\section{Data Availability}

The data of the points on the tool faces calculated in the example in Section 6 are used to support the findings of this study. These data are available from the corresponding author upon request.

\section{Conflicts of Interest}

The authors declare that there are no conflicts of interest regarding the publication of this paper.

\section{Acknowledgments}

This article was funded by the Natural Science Foundation of Tianjin (grant no. 18JCQNJC75200) and Tianjin Jingcheng Machine Tool Co. Ltd. (grant no. HX200134).

\section{References}

[1] D. Spath and A. Huhsam, "Skiving for high-performance machining of periodic structures," Annals of the CIRP, vol. 51, no. 1, pp. 472-475, 2002.

[2] J. Li, X. C. Chen, and H. Y. Zhang, "Slicing technology for cylindrical gears," Journal of Mechanical Engineering, vol. 47, no. 19, pp. 193-198, 2011.

[3] E. Guo, R. Hong, X. Huang, and C. Fang, "A novel power skiving method using the common shaper cutter," The International Journal of Advanced Manufacturing Technology, vol. 83, no. 1-4, pp. 157-165, 2016.

[4] H. Stadtfeld, "Power skiving of cylindrical gears on different machine platforms," Gear Technology, vol. 31, no. 1, pp. 52-62, 2014.

[5] C. Kobialka, "Contemporary gear pre-machining solutions," Gear Solutions, pp. 43-49, 2013.

[6] X. Chen, J. Li, B. Lou, J. Shi, and Q. Yang, "Effect of the cutter parameters and machining parameters on the interference in gear slicing," Chinese Journal of Mechanical Engineering, vol. 26, no. 6, pp. 1118-1126, 2013.
[7] J. Li, P. Wang, X.-C. Chen, and T.-J. Yang, "A study on the optimal selection of spur slice cutter parameters and machining parameters," The International Journal of Advanced Manufacturing Technology, vol. 82, no. 1-4, pp. 407-417, 2016.

[8] E. Guo, R. Hong, X. Huang, and C. Fang, "Research on the design of skiving tool for machining involute gears," Journal of Mechanical Science and Technology, vol. 28, no. 12, pp. 5107-5115, 2014.

[9] E. Guo, R. Hong, X. Huang, and C. Fang, "A correction method for power skiving of cylindrical gears lead modification," Journal of Mechanical Science and Technology, vol. 29, no. 10, pp. 4379-4386, 2015.

[10] K. Uriu, T. Osafune, T. Murakami et al., "Effects of shaft angle on cutting tool parameters in internal gear skiving," Journal of Mechanical Science and Technology, vol. 31, no. 12, pp. 5665-5673, 2017.

[11] C.-Y. Tsai, "Mathematical model for design and analysis of power skiving tool for involute gear cutting," Mechanism and Machine Theory, vol. 101, pp. 195-208, 2016.

[12] C.-H. Chen, "Geodesic relative curvature of instantaneous conjugation curve on base surface and geometrical differentials of third order," Mechanism and Machine Theory, vol. 43, no. 1, pp. 95-103, 2008.

[13] C.-H. Chen, "Geometrical way for describing body motion and equations of relationships between geometrical and kinematical parameters," Mechanism and Machine Theory, vol. 41, no. 3, pp. 283-306, 2006.

[14] K. Jia, S. Zheng, J. Guo, and J. Hong, "A surface envelopingassisted approach on cutting edge calculation and machining process simulation for skiving," The International Journal of Advanced Manufacturing Technology, vol. 100, no. 5-8, pp. 1635-1645, 2019.

[15] Y. P. Shih and Y. J. Li, "A novel method for producing a conical skiving tool with error-free flank faces for internal gear manufacture," Journal of Mechanical Design, vol. 140, no. 4, Article ID 043302, 2018.

[16] E. Guo, N. Ren, X. Ren, and C. Liu, “An efficient tapered tool having multiple blades for manufacturing cylindrical gears with power skiving," The International Journal of Advanced Manufacturing Technology, vol. 102, no. 9-12, pp. 2823-2832, 2019.

[17] Z. Guo, S.-M. Mao, L. Huyan, and D.-S. Duan, "Research and improvement of the cutting performance of skiving tool," Mechanism and Machine Theory, vol. 120, pp. 302-313, 2018.

[18] J. Li, P. Wang, Y. Q. Jin, Q. Hu, and X. C. Chen, "Cutting force calculation for gear slicing with energy method," International Journal of Advanced Manufacturing Technology, vol. 83, no. 58, pp. 887-896, 2016.

[19] I. Moriwaki, T. Osafune, M. Nakamura et al., "Cutting tool parameters of cylindrical skiving cutter with sharpening angle for internal gears," Journal of Mechanical Design, vol. 139, no. 3, Article ID 033301, 2017.

[20] N. Tapoglou, "Calculation of non-deformed chip and gear geometry in power skiving using a CAD-based simulation," The International Journal of Advanced Manufacturing Technology, vol. 100, no. 5-8, pp. 1779-1785, 2019.

[21] T. Tachikawa, D. Iba, N. Kurita, M. Nakamura, and I. Moriwaki, "Basic study on calculation of cutting forces useful for reducing vibration in skiving," Journal of Mechanical Design, vol. 139, no. 10, Article ID 104501, 2017.

[22] X. C. Chen, J. Li, Y. Zou, and P. Wang, "A study on the grinding of the major flank face of error-free spur slice cutter," International Journal of Advanced Manufacturing Technology, vol. 72, no. 1-4, pp. 425-438, 2014. 